\title{
Idade Relativa e Tempo de Passagem para Eremanthus incanus (Less.) Less em Uma Área em Recuperação no Município de Diamantina, MG
}

\author{
Lidia Santos $^{1}$, Marcio Oliveira ${ }^{1}$, Gilciano Nogueira ${ }^{1}$, Israel Pereira ${ }^{1}$, \\ Michele Silva ${ }^{1}$ \\ ${ }^{1}$ Departamento de Engenharia Florestal, Universidade Federal dos Vales do Jequitinhonha e Mucuri - UFVJM, \\ Diamantina/MG, Brasil
}

\begin{abstract}
RESUMO
O objetivo deste estudo foi determinar a idade relativa e o tempo de passagem dos indivíduos de Eremanthus incanus presente em uma área em recuperação, com avaliações periódicas ao longo de 4 anos. Os dados utilizados foram coletados na cidade de Diamantina, MG, por meio de inventários florestais contínuos em uma área de aproximadamente 2,15 hectares, totalizando 9 inventários florestais, os quais foram agrupados em classe de diâmetro de 3 e $5 \mathrm{~cm}$. Foram testados três modelos para estimar o incremento periódico médio mensal em diâmetro por centro de classe diamétrica. Através da melhor equação foram calculados a idade relativa e o tempo de passagem. Todos os três modelos ajustados foram melhores com a amplitude de classe de $3 \mathrm{~cm}$. A idade relativa e o tempo de passagem para os indivíduos de E. incanus alcançarem a menor classe de diâmetro foram de 4 e 2 anos, respectivamente.
\end{abstract}

Palavras-chave: incremento periódico, manejo florestal, centro de classe diamétrica.

\section{Relative Age and Passage Time for Eremanthus incanus (Less.) Less in A Recovery Area in the Municipality of Diamantina-MG}

\begin{abstract}
This study aimed to determine the relative age and the passage time of Eremanthus incanus individuals present in a recovery area, with periodic evaluations over four years. Data were collected in the city of Diamantina-MG, through continuous forest inventories in an area of 2.0 hectares, totaling nine forest inventories, which were grouped in 3 to $5 \mathrm{~cm}$ diameter classes. Three models were tested to estimate the monthly diameter average periodic increment by diameter class center. Relative age and passage time were calculated based in the best equation. All three adjusted models were better with the $3 \mathrm{~cm}$ class range. The relative age and the passage time for $E$. incanus individuals to achieve the smallest class size was 4 and 2 years, respectively.
\end{abstract}

Keywords: periodic increment, forest management, diameter class center. 


\section{INTRODUÇÃO}

Eremanthus incanus (Less.) Less conhecida popularmente como candeia, comum nos estados de Minas Gerais e Bahia, tem sido utilizada para a produção de moirões e na farmacologia. Capaz de se desenvolver em áreas degradadas, se destaca dentre as poucas espécies que conseguem sobreviver nessas áreas (Scolforo et al., 2012). Dentre as espécies florestais brasileiras de múltiplo uso, os indivíduos de E. incanus têm se destacado pela sua rusticidade, tornando imprescindível a realização do manejo florestal (Ferreira et al., 2014).

Para elaboração de um plano de manejo aplicado a espécies nativas sobre as quais há escassez de informação é necessário a realização do inventário florestal contínuo. Por meio desses inventários são adquiridas informações relacionadas à quantidade, qualidade e condição dos recursos florestais (Husch et al., 2003).

Uma informação importante, mas de difícil obtenção em espécies ou florestas nativas, é a idade, visto que as árvores apresentam idades diferentes (Imaña Encinas et al., 2005). Para o manejo florestal sustentável, a possibilidade de estimar a idade das árvores é fundamental, pois através dela é possível determinar o ciclo de corte, o volume, planejar as intervenções silviculturais, entre outros. Entre possíveis técnicas utilizadas para estimar a idade destacam-se: análise do tronco, contagem dos verticilos e anéis de crescimento. Entretanto, dependendo das espécies estudadas, essas técnicas podem não ser recomendadas (Avery \& Burkhart, 2002).

Uma alternativa para determinar a idade foi proposta por Caballero \& Malleux (1976), os quais para avaliar a idade relativa de uma espécie ou povoamentos nativos utilizaram a estimação do crescimento periódico do diâmetro pelo número de árvores por classe diamétrica encontrado nos inventários florestais sucessivos. Essa metodologia foi aplicada por vários autores, como Del Valle (1986), Araújo et al. (1993), Souza et al. (1993), Scolforo et al. (1996) e Silva et al. (1999), entre outros. De acordo com Souza et al. (1993), essa metodologia assume que o tamanho das árvores é função da sua idade, ou seja, o crescimento do diâmetro da árvore é diretamente proporcional a sua idade.

A partir da determinação das idades relativas de uma floresta é possível, por meio do método de tempo de passagem, quantificar o período necessário para que os indivíduos da floresta mudem de classe diamétrica (Caballero \& Malleux, 1976), podendo-se definir o ciclo de corte, além de conhecer a evolução do número de árvores por classe de diâmetro ao longo do tempo, o que proporciona informações sobre a produção sustentável das florestas naturais (Araujo et al., 1993).

A metodologia da idade relativa e o tempo de passagem foi aplicada na maioria das vezes em florestas de porte maior, sendo escassos os trabalhos em áreas de formação mais inicial como áreas em processo de recuperação. Sendo assim, o objetivo do trabalho foi determinar a idade relativa e o tempo de passagem dos indivíduos de Eremanthus incanus presentes em uma área em processo de recuperação em Diamantina, MG.

\section{MATERIAL E MÉTODOS}

A área de estudo localiza-se em um antigo depósito de lixo da cidade de Diamantina em Minas Gerais, que segundo Machado et al. (2012) foi isolada em 2002 para fins de restauração. Encontra-se situada nas coordenadas $18^{\circ} 12^{\prime} 17^{\prime \prime}$ de latitude Sul e $43^{\circ} 34^{\prime} 08^{\prime \prime}$ de longitude Oeste, a uma altitude de aproximadamente $1.400 \mathrm{~m}$, presente no Campus JK da Universidade Federal dos Vales do Jequitinhonha e Mucuri, região do Alto Jequitinhonha, no Complexo da Serra do Espinhaço.

O regime climático da região é tipicamente tropical, Cwb na classificação de Köppen, caracterizado por verões brandos e úmidos nos meses de outubro a abril e invernos mais frescos e secos nos meses de junho e agosto, com precipitação pluviométrica média anual variando de 1.250 a $1.550 \mathrm{~mm}$ e temperatura média anual na faixa de $18^{\circ} \mathrm{C}$ a $19^{\circ} \mathrm{C}$, sendo predominantemente amenas durante todo o ano (Neves et al., 2005).

$\mathrm{O}$ presente estudo foi realizado em uma área de aproximadamente 2,15 hectares (Figura 1).

Os inventários florestais foram realizados entre setembro de 2010 e setembro de 2014, totalizando nove inventários: o $1^{\circ}$ obtido em setembro de 2010; o $2^{\circ}$, em fevereiro de 2011; o $3^{\circ}$, em junho de 2011; o $4^{\circ}$, em setembro de 2011; o $5^{\circ}$, em fevereiro de 2012; o $6^{\circ}$, em setembro de 2012; o $7^{\circ}$, em maio de 2013; o $8^{\circ}$, em janeiro de 2014; e $9^{\circ}$, em setembro de 2014.

Os inventários florestais foram realizados por meio do censo dos indivíduos de E. incanus na área de estudo, de forma que todos os indivíduos vivos com diâmetro 


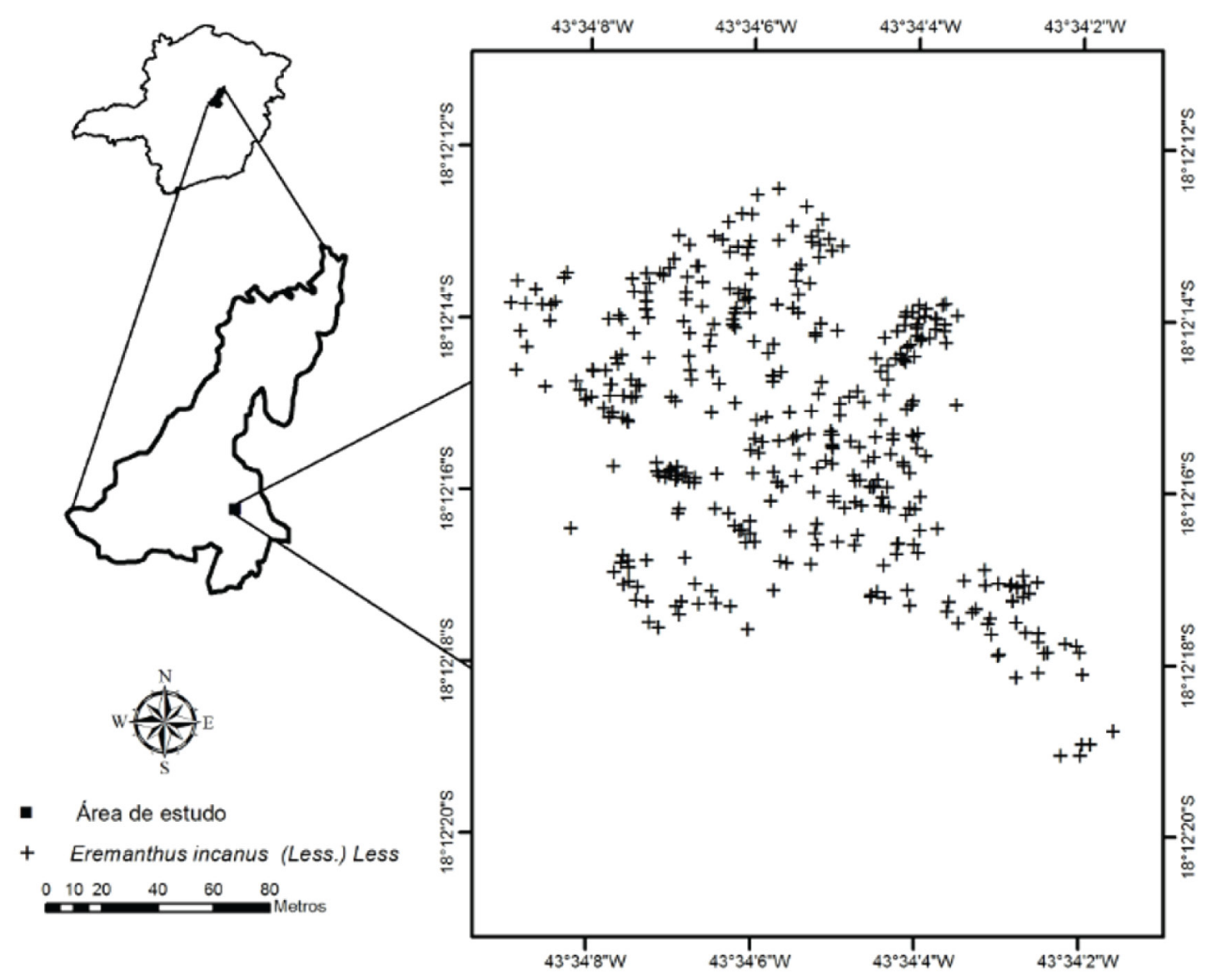

Figura 1. Área de estudo em recuperação no antigo lixão da cidade de Diamantina, MG.

Figure 1. Study area under recovering process of the old waste dump at Diamantina city, MG.

a $0,3 \mathrm{~m}$ do solo (DAS) $\geq$ a 5,0 $\mathrm{cm}$ foram mensurados. Entretanto, como a árvore de candeia pode apresentar vários fustes, o critério de inclusão utilizado foi que pelo menos um dos fustes do indivíduo tivesse DAS $\geq$ a $5,0 \mathrm{~cm}$.

A variável dendrométrica mensurada nos inventários foi a circunferência a $0,3 \mathrm{~m}$ de altura do solo (CAS). Os dados de circunferência foram coletados com fita métrica e todas as árvores foram georreferenciadas e identificadas com placas de alumínio numeradas.

Para determinar a idade relativa e o tempo de passagem por classe diamétrica dos indivíduos de E. incanus utilizou-se a metodologia de Caballero \& Malleux (1976), que permite estimar a idade relativa de uma floresta por meio da correlação entre o centro de classe diamétrica e o incremento periódico médio em diâmetro, a partir de dados provenientes de inventários florestais em parcelas permanentes. Assim, com os DAS dos indivíduos de E. incanus determinou-se o incremento periódico médio mensal (IPM) de cada árvore para os intervalos de medição 4, 8 e 12 meses. Para aplicar os três intervalos de medição utilizaram-se os inventários realizados na área, os quais foram relacionados em pares para cada intervalo de medição, sempre que possível.

Os valores encontrados de IPM para cada período de intervalo de medição foram agrupados por classes de diâmetro. Para analisar a amplitude de classe diamétrica que melhor explicasse a distribuição da espécie testaram-se duas amplitudes de classe de diâmetro, de $3 \mathrm{~cm}$ e $5 \mathrm{~cm}$, para agrupar os dados de IPM. Em seguida, com os dados de IPM agrupados com os respectivos centros de classes diamétricas, ajustaram-se equações, utilizando-se os modelos lineares simples, Equação 1, o quadrático, Equação 2, e o modelo não linear logístico, Equação 3, os quais foram ajustados no software R Development Core Team (2013) pela 
função $\operatorname{lm}()$ para os modelos lineares e pela função nls() para o modelo não linear:

$$
\begin{aligned}
& Y_{i}=\beta_{0}+\beta_{1} X_{i}+\varepsilon \\
& Y_{i}=\beta_{0}+\beta_{1} X_{i}+\beta_{2} X_{i}^{2}+\varepsilon \\
& Y_{i}=\frac{\beta_{0}}{1+\beta_{1} e^{-\beta_{2} X_{i}}} \varepsilon
\end{aligned}
$$

em que: $Y_{i}=$ incremento periódico médio mensal em diâmetro da classe diâmetrica i $(\mathrm{cm} / \mathrm{mês}) ; X_{i}=$ centro de classe diamétrica $\mathrm{i}$, em $\mathrm{cm} ; \beta_{0}, \beta_{1}$ e $\beta_{2}=$ parâmetros a serem estimados; $\mathcal{E}=$ erro aleatório para $\mathrm{i}=1,2, \ldots, \mathrm{n}$ classe de diâmetro.

A escolha da equação que melhor relacionou o IPM de diâmetro com o centro de classe diamétrica foi realizada pela análise do coeficiente de correlação $\left(r_{x y}\right)$, erro padrão $\left(S_{x y}\right)$ e gráfico de resíduo para todas as equações. As equações também foram comparadas por meio do Critério de Informação de Akaike (AIC), usado para selecionar modelos e que se baseia no máximo da função de verossimilhança, uma vez que o modelo que apresentar o menor valor de AIC é considerado o modelo de melhor ajuste (Akaike, 1974). O método para estimar o melhor modelo é dado pela Equação 4:

$$
A I C=-2 \ln (m v)+2 p
$$

em que: $\ln =$ logaritmo na base neperiana; $m v$ = máximo valor da função de verossimilhança;

$p=$ número de parâmetros do modelo.

Por meio das melhores equações para os períodos de intervalo de medição analisados em cada amplitude de classe testada estimou-se o IPM diamétrico. O primeiro IPM estimado foi calculado utilizando-se o menor DAS $(5 \mathrm{~cm})$ dos indivíduos de E. incanus mensurados, pois de acordo com Caballero \& Malleux (1976), nos primeiros anos o incremento diamétrico se assemelha ao de menor diâmetro. Os DAS seguintes foram determinados sucessivamente pela soma do DAS anterior com o seu IPM estimado.

Com os valores de DAS e IPM estimado realizou-se o cálculo da primeira idade relativa por meio da divisão do menor DAS mensurado na área $(5 \mathrm{~cm})$ pelo primeiro IPM estimado. De acordo com Caballero \& Malleux (1976), essa idade representa a idade necessária para uma árvore ingressar na primeira classe diamétrica. Para o cálculo das idades relativas seguintes, realizou-se a soma de 1 ano para cada IPM estimado, tendo a primeira idade relativa como base.
O tempo de passagem entre as classes diamétricas foi encontrado por meio da diferença entre as menores idades relativas correspondentes a duas classes diamétricas consecutivas.

Todas as análises realizadas neste estudo foram através do software R Development Core Team (2013), com apoio da plataforma RStudio versão 0.97.551.

\section{RESULTADOS}

Foram encontrados 190, 205, 232, 233, 270, 307, 339, 344 e 364 indivíduos de E. incanus nos nove inventários florestais listados, respectivamente. Os incrementos periódicos médios (IPM) em diâmetro obtidos nos períodos de intervalos de medição de 4, 8 e 12 meses, os quais estiveram agrupados em classe de diâmetro de $3 \mathrm{~cm}$ e $5 \mathrm{~cm}$, estão representados na Tabela 1 . A partir dos resultados observou-se que o IPM do diâmetro

Tabela 1. Incremento periódico médio (IPM) em diâmetro por centro de classe diamétrica para a espécie Eremanthus incanus, com amplitudes de classe de diâmetro de $3 \mathrm{~cm}$ e $5 \mathrm{~cm}$ e períodos de intervalo de medição de 4, 8 e 12 meses, realizadas em uma área em Diamantina, MG.

Table 1. Mean Periodic Increment (MPI) in diameter per diameter class center for Eremanthus incanus

\begin{tabular}{|c|c|c|c|c|}
\hline \multirow{3}{*}{$\begin{array}{l}\text { Amplitude } \\
\text { de classe } \\
(\mathrm{cm})\end{array}$} & \multirow{3}{*}{$\begin{array}{l}\text { Centro } \\
\text { de classe } \\
(\mathrm{cm})\end{array}$} & \multicolumn{3}{|c|}{$\begin{array}{c}\text { Período de intervalo de } \\
\text { medição (mês) }\end{array}$} \\
\hline & & 4 & 8 & 12 \\
\hline & & IPM & IPM & IPM \\
\hline \multirow{10}{*}{3} & 6,5 & 0,1321 & 0,0910 & 0,0928 \\
\hline & 9,5 & 0,1839 & 0,1055 & 0,1357 \\
\hline & 12,5 & 0,1721 & 0,1230 & 0,1369 \\
\hline & 15,5 & 0,2264 & 0,1380 & 0,1496 \\
\hline & 18,5 & 0,2393 & 0,1500 & 0,1704 \\
\hline & 21,5 & 0,2773 & 0,1671 & 0,1631 \\
\hline & 24,5 & 0,2540 & 0,1850 & 0,1391 \\
\hline & 27,5 & 0,4011 & 0,2298 & 0,1726 \\
\hline & 30,5 & 0,4962 & 0,2549 & \\
\hline & 33,5 & 0,4271 & & \\
\hline \multirow{6}{*}{5} & 7,5 & 0,1509 & 0,1030 & 0,1134 \\
\hline & 12,5 & 0,1847 & 0,1272 & 0,1414 \\
\hline & 17,5 & 0,2339 & 0,1436 & 0,1597 \\
\hline & 22,5 & 0,2729 & 0,1646 & 0,1480 \\
\hline & 27,5 & 0,4487 & 0,1967 & 0,1726 \\
\hline & 32,5 & 0,4271 & 0,2549 & \\
\hline
\end{tabular}
species, with diameter class widths of 3 and $5 \mathrm{~cm}$ and measured period with interval of 4,8 and 12 months, carried out in an area in Diamantina-MG. 
diminui com o aumento do intervalo de tempo entre as medições para as duas amplitudes de classe utilizadas. No intervalo de medição de 4 meses, o incremento médio foi de $0,84 \mathrm{~cm} \cdot \mathrm{ano}^{-1}$, variando de $0,39 \mathrm{~cm}$ a $1,49 \mathrm{~cm} \cdot$ ano $^{-1}$. Para os períodos de intervalo de medição de 8 e 12 meses o incremento médio foi de $0,24 \mathrm{~cm} \mathrm{e}$ $0,14 \mathrm{~cm}$.ano ${ }^{-1}$, variando de $0,14 \mathrm{~cm}$ a $0,38 \mathrm{~cm} \cdot$ ano $^{-1} \mathrm{e}$ de $0,09 \mathrm{~cm}$ a $0,17 \mathrm{~cm} \cdot$ ano $^{-1}$, respectivamente.

Com base nos resultados de IPM de diâmetro por centro de classe diamétrica encontrados foi possível estimar o IPM de diâmetro utilizando-se os modelos lineares e o modelo não linear (Tabela 2). Analisando-se o coeficiente de correlação e o erro padrão da média para os três intervalo de medição utilizados observaram-se resultados próximos para todos. Com intuito de avaliar os modelos, utilizou-se o método da análise do critério de AIC, o qual demonstrou que o melhor intervalo de medição foi o de 8 meses para os três modelos avaliados na amplitude de classe diamétrica de $3 \mathrm{~cm}$. A amplitude de classe de diâmetro de $3 \mathrm{~cm}$ foi a que melhor explicou o IPM de diâmetro, pois apresentou melhores resultados para os três modelos ajustados.
A partir dos modelos ajustados foram elaborados os gráficos de resíduo para todos os períodos de intervalos de medição e amplitude de classe diamétrica estudados (Figura 2). De acordo com os gráficos, os três modelos utilizados apresentaram uma distribuição semelhante para cada período de intervalo de medição e amplitude de classe de diâmetro, sendo que para o período de intervalo de medição de 4 meses a amplitude de $3 \mathrm{~cm}$ apresentou melhor ajuste dos dados para os três modelos. No período de intervalo de medição de 8 meses, a classe de diâmetro de $5 \mathrm{~cm}$ foi a que apresentou melhor ajuste dos dados. Já no intervalo de medição 12 meses, as duas classes de diâmetro avaliadas foram satisfatórias. Entre os três períodos de intervalo de medição analisados, o de 12 meses foi o que apresentou melhor ajuste dos dados.

As estimativas da idade relativa e do tempo de passagem para a amplitude de classe de diâmetro de $3 \mathrm{~cm}$ e respectivos períodos de intervalo de medição (4, 8 e 12 meses) estão apresentadas na Tabela 3. Para o período de intervalo de medição de 4 meses, o melhor modelo foi o linear quadrático, para o intervalo de

Tabela 2. Resultados dos coeficientes $\left(\beta_{n}\right)$ gerados pelos três modelos ajustados, com respectivos coeficientes de correlação $\left(r_{x y}\right)$, erro padrão da média $\left(s_{x y}\right)$ e Critério de Akaike (AIC) em diferentes intervalos de medições (IIF) e amplitudes de classes para a espécie Eremanthus incanus, em Diamantina, MG.

Table 2. Results of the coefficients $\left(\beta_{n}\right)$ generated by the three fitted models with their respective coefficients of correlation $\left(r_{x y}\right)$, standard error of the mean $\left(s_{x y}\right)$ and Criterion of Akaike $(A I C)$ at different intervals measurements (IIF) and amplitude classes for Eremanthus incanus species, located in Diamantina-MG.

\begin{tabular}{|c|c|c|c|c|c|c|c|c|}
\hline IIF & $\begin{array}{l}\text { Amplitude } \\
\text { de classe }\end{array}$ & Modelo & $\boldsymbol{\beta}_{0}$ & $\boldsymbol{\beta}_{1}$ & $\boldsymbol{\beta}_{2}$ & $r_{x y}$ & $s_{x y}$ & AIC \\
\hline \multirow{6}{*}{4} & \multirow{3}{*}{3} & 1 & 0,0692 & 0,0106 & - & 0,834 & 1,054 & $-72,22$ \\
\hline & & 2 & 0,1566 & $-0,0007$ & 0,0003 & 0,858 & 1,052 & $-73,78$ \\
\hline & & 3 & $-1,0203$ & $-9,1794$ & 0,0319 & 0,857 & 1,052 & $-73,59$ \\
\hline & \multirow{3}{*}{5} & 1 & 0,0487 & 0,0113 & - & 0,829 & 1,060 & $-44,40$ \\
\hline & & 2 & 0,1245 & 0,0014 & 0,0003 & 0,844 & 1,060 & $-43,82$ \\
\hline & & 3 & 1,9757 & 18,6363 & 0,0569 & 0,842 & 1,060 & $-43,74$ \\
\hline \multirow{6}{*}{8} & \multirow{3}{*}{3} & 1 & 0,0482 & 0,0059 & - & 0,639 & 1,049 & $-158,20$ \\
\hline & & 2 & 0,0802 & 0,0013 & 0,0001 & 0,646 & 1,049 & $-157,00$ \\
\hline & & 3 & $-0,2965$ & $-4,8883$ & 0,0268 & 0,647 & 1,049 & $-157,17$ \\
\hline & \multirow{3}{*}{5} & 1 & 0,0787 & 0,0043 & - & 0,568 & 1,048 & $-97,56$ \\
\hline & & 2 & 0,0947 & 0,0007 & 0,0001 & 0,573 & 1,051 & $-102,44$ \\
\hline & & 3 & $-0,3799$ & $-5,3404$ & 0,0224 & 0,576 & 1,050 & $-101,33$ \\
\hline \multirow{6}{*}{12} & \multirow{3}{*}{3} & 1 & 0,0964 & 0,0030 & - & 0,658 & 1,023 & $-91,55$ \\
\hline & & 2 & 0,0285 & 0,0129 & $-0,0003$ & 0,780 & 1,019 & $-96,95$ \\
\hline & & 3 & 0,1587 & 6,1733 & 0,3414 & 0,790 & 1,019 & $-97,78$ \\
\hline & \multirow{3}{*}{5} & 1 & 0,1036 & 0,0026 & - & 0,731 & 1,016 & $-67,02$ \\
\hline & & 2 & 0,0672 & 0,0077 & $-0,0002$ & 0,782 & 1,015 & $-67,36$ \\
\hline & & 3 & 0,1584 & 2,8575 & 0,2624 & 0,805 & 1,015 & $-68,64$ \\
\hline
\end{tabular}



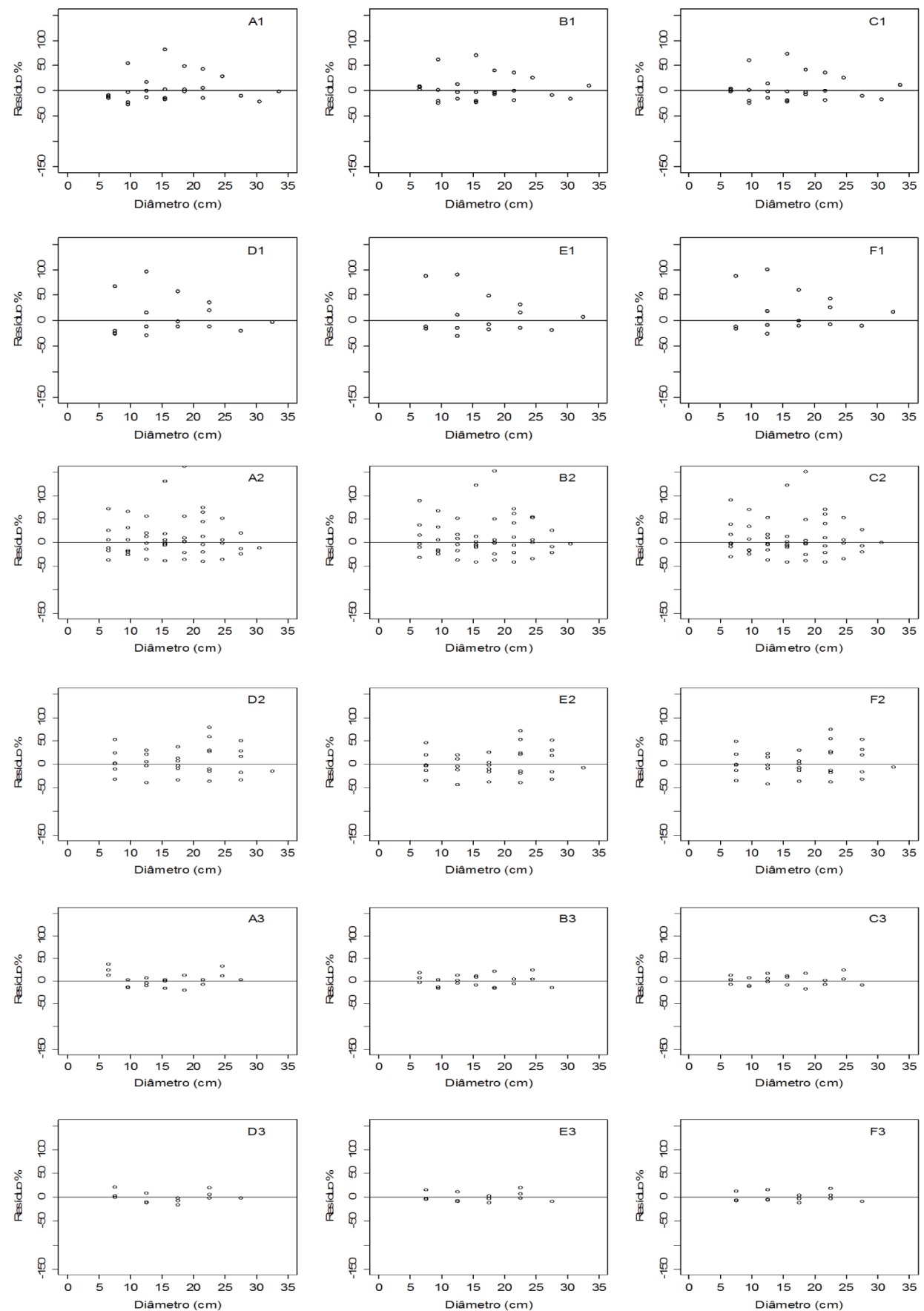

Figura 2. Tendência do incremento periódico em diâmetro (IPM) por classe de DAS para os indivíduos Eremanthus incanus localizados na cidade de Diamantina, MG. A-C são os modelos 1, 2 e 3, respectivamente, na amplitude de classe de $3 \mathrm{~cm}$; D-F são os modelos 1, 2 e 3, respectivamente, na amplitude de classe de $5 \mathrm{~cm}$; e os números 1,2 e 3 junto às letras utilizadas para identificação dos gráficos são os períodos de intervalo de medição de 4,8 e 12 meses, respectivamente.

Figure 2. Tendency of the mean periodic increment (MPI) in diameter per DAS class for Eremanthus incanus individuals, located in the city of Diamantina, Minas Gerais. Where: A-C are the models 1, 2 and 3, respectively, at class widths of $3 \mathrm{~cm}$; D-F are the models 1, 2 e 3, respectively, at class widths of $5 \mathrm{~cm}$; and the numbers 1, 2 and 3 together with letters were used to identify the graphs, which are the measured period with intervals of 4,8 and 12 months respectively. 
Tabela 3. Idade relativa e tempo de passagem por classe de diâmetro para os períodos de intervalo de medição de 4, 8 e 12 meses, com amplitude de classe de $3 \mathrm{~cm}$, utilizando-se o modelo linear simples (1) e quadrático (2) e o modelo logístico (3) para a espécie Eremanthus incanus, em Diamantina, MG.

Table 3. Relative Age and Passing Time, per diameter class for the measured period with intervals of 4, 8 and 12 months with class width of $3 \mathrm{~cm}$, using the simple linear model (1), the quadratic model (2), and the logistic one (3), for Eremanthus incanus species, located in Diamantina-MG.

\begin{tabular}{|c|c|c|c|c|c|c|}
\hline $\begin{array}{c}\text { Intervalo de } \\
\text { medição }(\mathrm{cm})\end{array}$ & Modelo & $\begin{array}{l}\text { Centro de } \\
\text { classe }(\mathrm{cm})\end{array}$ & DAS (cm) & IPM $(\mathbf{c m})$ & Idade (anos) & $\begin{array}{c}\text { Tempo de } \\
\text { passagem (mês) }\end{array}$ \\
\hline \multirow{10}{*}{4} & \multirow{10}{*}{2} & 6,5 & 6,96 & 0,1664 & 3 & 18 \\
\hline & & 9,5 & 9,00 & 0,1748 & 4 & 17 \\
\hline & & 12,5 & 11,16 & 0,1865 & 5 & 15 \\
\hline & & 15,5 & 13,48 & 0,2022 & 6 & 15 \\
\hline & & 18,5 & 16,01 & 0,2232 & 7 & 12 \\
\hline & & 21,5 & 18,83 & 0,2510 & 8 & 9 \\
\hline & & 24,5 & 22,04 & 0,2886 & 9 & 9 \\
\hline & & 27,5 & 25,77 & 0,3401 & 10 & 9 \\
\hline & & 30,5 & 30,23 & 0,4128 & 11 & 9 \\
\hline & & 33,5 & 35,72 & 0,5190 & 12 & 6 \\
\hline \multirow{10}{*}{8} & \multirow{10}{*}{1} & 9,5 & 8,13 & 0,1408 & 4 & 25 \\
\hline & & 12,5 & 11,27 & 0,1764 & 5 & 20 \\
\hline & & 15,5 & 14,35 & 0,2113 & 6 & 16 \\
\hline & & 18,5 & 17,04 & 0,2419 & 7 & 12 \\
\hline & & 21,5 & 20,14 & 0,2769 & 8 & 12 \\
\hline & & 24,5 & 23,05 & 0,3099 & 9 & 10 \\
\hline & & 28,5 & 26,31 & 0,3469 & 10 & 10 \\
\hline & & 30,5 & 29,20 & 0,3796 & 11 & 8 \\
\hline & & 33,5 & 32,36 & 0,4154 & 12 & 8 \\
\hline & & 36,5 & 34,92 & 0,4444 & 13 & 6 \\
\hline \multirow{10}{*}{12} & \multirow{10}{*}{3} & 9,5 & 8,08 & 0,1141 & 5 & 32 \\
\hline & & 12,5 & 11,13 & 0,1396 & 6 & 24 \\
\hline & & 15,5 & 14,05 & 0,1511 & 7 & 20 \\
\hline & & 18,5 & 17,12 & 0,1561 & 8 & 20 \\
\hline & & 21,5 & 20,11 & 0,1578 & 9 & 19 \\
\hline & & 24,5 & 23,11 & 0,1585 & 10 & 19 \\
\hline & & 27,5 & 26,13 & 0,1587 & 11 & 19 \\
\hline & & 30,5 & 29,14 & 0,1588 & 12 & 19 \\
\hline & & 33,5 & 32,00 & 0,1588 & 13 & 18 \\
\hline & & 36,5 & 35,02 & 0,1588 & 14 & 19 \\
\hline
\end{tabular}

8 meses, o linear simples e para o intervalo de 12 meses, o logístico. O resultado demonstra que os três modelos podem ser utilizados para calcular a idade relativa e o tempo de passagem da espécie E. incanus, dependendo do período de intervalo de medição utilizado.

Entre os períodos de intervalo de medições analisados pode-se observar que a idade relativa e o tempo de passagem aumentaram com o aumento do intervalo de medição, ou seja, o intervalo de 12 meses foi o que apresentou idades relativas maiores. Já o período de medição de 4 meses utilizando o modelo linear quadrático ajustado foi o que apresentou a menor idade relativa e tempo de passagem.

As menores idades relativas iniciais estimadas foram de 3, 4 e 5 anos, com tempo de passagem de 18, 25 e 32 meses para os indivíduos de E. incanus medidos em um período de intervalo de 4, 8 e 12 meses, respectivamente. A menor idade relativa encontrada por período de intervalo de medição representa o número de anos para que os indivíduos de E. incanus em regeneração ingressem no tamanho mínimo de diâmetro estudado, que nesse caso foi de $5 \mathrm{~cm}$. 
O tempo de passagem para as classes de diâmetro menor foi maior que o das classes maiores e o IPM diamétrico aumentou com o aumento das classes diamétricas, o que demonstra a relação do incremento diamétrico com o tempo de passagem, ou seja, quanto maior o IPM menor será o tempo de passagem.

O uso do método da análise da idade relativa e tempo de passagem entre as classes diamétricas conseguiram estimar idades para os indivíduos de E. incanus na área estudada.

\section{DISCUSSÃO}

A diminuição do incremento diamétrico ocorreu com o aumento da idade dos indivíduos, uma vez que ao se utilizar um intervalo de medição de 4 meses o IPM do diâmetro foi maior em relação ao intervalo de 8 e de 12 meses, ou seja, essa diminuição ocorreu devido ao aumento do intervalo entre as medições. O incremento periódico diamétrico dos indivíduos de E. Incanus, segundo Araújo et al. (2014), diminui com o aumento da idade, mostrando ainda a influência do efeito densidade de plantas sobre o diâmetro.

A possibilidade de utilizar o período de medição inadequado deve ser levada em consideração ao se estudar os indivíduos de E. incanus e estudos mais detalhados devem ser realizados. Souza et al. (1993), trabalhando com povoamentos florestais tropicais nativos, utilizaram um intervalo de medição de 9 anos para determinar o incremento diamétrico e, dentro desse intervalo, calcularam os incrementos periódicos médios anuais para três medições, com períodos de intervalo de medição de 3 anos cada e, em seguida, comparando os resultados encontrados, observaram diferenças, já que para esse tipo de floresta intervalos de medição menores que 5 anos podem ocasionar erros. Dessa forma, o período de intervalo de medição de 4 meses, que apresentou o IPM maior em relação aos intervalos de 8 e de 12 meses, pode não ser necessariamente o que melhor representa a área, pois esse intervalo pode ser pequeno para a espécie em estudo, causando uma superestimação dos dados de IPM.

Ao se estimar o IPM de diâmetro para o E. incanus observou-se que o modelo linear simples, o modelo linear quadrático e o modelo logístico apresentaram melhores resultados no período de intervalo de medição de 8, 4 e 12 meses, respectivamente. Os três modelos apresentaram uma distribuição, nos gráficos de resíduos, muito semelhante. O modelo logístico, por possuir melhor fundamentação biológica, geralmente é menos sensível às particularidades dos dados utilizados no ajuste, apresentando resultados melhores (Batista et al., 2001), como observado no intervalo de medição de 12 meses. No entanto, nos períodos de intervalo de 4 e de 8 meses, os modelos lineares quadrático e simples foram melhor, respectivamente, sendo que um aspecto importante a ser considerado nesses modelos é a facilidade de ajustá-los.

A amplitude de classe $3 \mathrm{~cm}$ apresentou resultados melhores comparativamente à amplitude de $5 \mathrm{~cm}$, uma vez que com um número de classes de diâmetro maior a distribuição dos indivíduos dentro dessas classes torna-se mais representativa, diminuindo a generalização do processo dinâmico dentro das classes diamétricas. Scolforo et al. (2012) utilizaram amplitude de classe de diâmetro de $3 \mathrm{~cm}$ para avaliar a distribuição do número de árvores de E. incanus por classe diamétrica em estrato arbóreo no município de Morro do Pilar, Minas Gerais, no entanto, ao analisarem as taxas de crescimento médio e acelerado, a amplitude de classe de diâmetro escolhida foi a de $5 \mathrm{~cm}$. Assim, a ausência de padronização da amplitude de classe diamétrica a ser utilizado ao se trabalhar com o E. incanus é comum e, de acordo com os resultados deste trabalho, a amplitude de classe diamétrica de $3 \mathrm{~cm}$ é ideal para a espécie.

Analisando-se duas amplitudes de classe diferentes em uma Floresta Ombrófila Mista, Ebling et al. (2012) observaram que a classe diamétrica com a amplitude maior apresenta estimativas menos precisas. No entanto, esses resultados foram para uma local com espécies diferentes da estudada neste trabalho, o que não impede a discussão, uma vez que a escolha do tamanho da amplitude de classe diamétrica pode influenciar no estudo de qualquer espécie.

As menores idades e tempo de passagem encontradas para os indivíduos de E. incanus foram utilizando-se o modelo linear quadrático ajustado no intervalo de medição de 4 meses, o modelo linear simples ajustado no intervalo de medição de 8 meses e o modelo logístico ajustado no intervalo de medição de 12 meses. Entretanto, o modelo linear foi considerado por Caballero \& Malleux (1976), Araujo et al. (1993), Souza et al. (1993) e Silva et al. (1999) como o melhor para a metodologia aplicada. Caballero \& Malleux 
(1976) constataram, ao testar as equações lineares simples e quadráticas em floresta natural, uma melhor eficiência da equação linear quadrática, utilizada para estimar o incremento e a idade das árvores estudadas. Araujo et al. (1993), testando diferentes modelos em povoamentos florestais nativos, selecionaram também uma equação linear quadrática para estimar o incremento periódico médio anual, a idade relativa e o tempo de passagem por classe diamétrica em função do diâmetro. Souza et al. (1993), analisando também um povoamento florestal nativo, utilizaram o modelo linear quadrático para estimar o incremento periódico médio anual e, em seguida, a idade relativa e o tempo de passagem por classe diamétrica.

A primeira idade definida em cada modelo escolhido foi entre 3 e 5 anos, conforme o intervalo de medição utilizado, sendo que essa é a idade necessária para um indivíduo de E. incanus conseguir alcançar o menor diâmetro $(5 \mathrm{~cm})$ e, consequentemente, ingressar na menor classe diamétrica. Estudos para os indivíduos de E. incanus analisando o DAS em vez do diâmetro a altura do peito (DAP) ainda são escassos e insuficientes para comparações mais apropriadas, visto que os trabalhos envolvendo a espécie utilizam geralmente o DAP.

Pérez et al. (2004), estudando o E. Erythropappus, encontraram o diâmetro na base da árvore de $7,5 \mathrm{~cm}$ na idade de 18 anos, no entanto, segundo os autores, a idade encontrada foi alta, não refletindo o tempo real devido à competição por luz com outras espécies e até mesmo outras candeias presentes no local, ocorrendo um crescimento mais lento. Dessa forma pode-se observar que a idade de ingresso para as espécies E. erythropappus ou E. incanus varia de acordo com o local, tornando os resultados encontrados fundamentais para embasar outros estudos.

O IPM do diâmetro estimado dos indivíduos de E. incanus aumentou com o avanço das idades encontradas. Essa característica pode ser observada em espécies exigentes quanto à luz, para as quais as condições ambientais em que a espécie se encontra apresentam relação direta com o crescimento (Condit et al., 1993). As classes diamétricas menores crescem mais lentamente que as maiores em floresta sem interferência e o incremento em diâmetro aumenta com o aumento das classes diamétricas (Souza et al., 1993). Araújo et al. (2014), ao estudarem o IPM de diâmetro do E. incanus encontraram resultados semelhantes aos encontrados neste trabalho, no entanto, os indivíduos analisados pelos autores são indivíduos mais velhos, os quais já tinham alcançado valor de IPM máximo. A maioria dos indivíduos de E. incanus presentes na área de estudo são indivíduos mais novos. Como os maiores IPM de diâmetro encontrados estão com idades em torno de 13 anos e ainda não alcançaram o IPM de diâmetro máximo, pode-se concluir que os indivíduos ainda não entraram em competição.

Em relação ao tempo de passagem, observou-se que os indivíduos nas menores classes de diâmetro precisam de mais tempo para mudar de classe. $\mathrm{O}$ método de tempo de passagem permite estimar a idade aproximada para o indivíduo estar em cada classe, consequentemente, determina o ciclo de corte (Silva et al., 1999). O método também contribui para a projeção futura para organização e produção sustentável de florestas naturais (Araujo et al., 1993).

O método da idade relativa e tempo de passagem apresenta potencial para contribuir nos estudos dos indivíduos de E. incanus.

\section{CONCLUSÃO}

A idade relativa e o tempo de passagem para os indivíduos de E. incanus alcançarem a menor classe de diâmetro foram de 4 e 2 anos, respectiva e aproximadamente. $\mathrm{O}$ melhor intervalo de medição para se utilizar no cálculo da idade relativa e tempo de passagem foi de 8 meses.

O tempo de passagem foi maior nas menores classes de diâmetro para os indivíduos de E. incanus.

\section{STATUS DA SUBMISSÃO}

Recebido: 20 abr., 2016

Aceito: 23 abr., 2016

\section{AUTOR(ES) PARA CORRESPONDÊNCIA}

\section{Lidia Santos}

Departamento de Engenharia Florestal, Universidade Federal dos Vales do Jequitinhonha e Mucuri - UFVJM, Rodovia MGT, Km 583, 5000, CEP 39100-000, Diamantina, MG, Brasil e-mail: lidiagabriellasantos@gmail.com 


\section{REFERÊNCIAS}

Akaike H. A new look at the statistical model identification. IEEE Transactions on automatic control 1974, 19(6): 716-723.

Araújo EJG, David HC, Morais VA, Scolforo JRS, Péllico S No, Zanette VH et al. Crescimento em diâmetro e altura da regeneração natural de Eremanthus incanus. Floresta 2014; 44(2): 217-228. http://dx.doi.org/10.5380/rf.v44i2.31362.

Araújo PA, Souza AL, Campos JCC, Paula F No. Idade relativa como subsídio à determinação de ciclo de corte no manejo sustentável de povoamentos florestais nativos. Revista Árvore 1993; 17(1): 100-116.

Avery TE, Burkhart HE. Forest measurements. 5th ed. New York: McGraw-Hill Higher Education; 2002.

Batista JLF, Couto HTZ, Marquesini M. Desempenho de modelos de relações hipsométricas: estudo em três tipos de floresta. Scientia Forestalis 2001; 60: 149-163.

Caballero JD, Malleux JO. Estudio de una metodologia para la determinación de edades en bosques naturales. Revista Floresta del Perú 1976; 5(1-2): 33-40.

Condit R, Hubbell SP, Foster RB. Identifying fast-growing native trees from the neotropics using data from a large, permanent census plot. Forest Ecology and Management 1993; 62(1-4): 123-143. http://dx.doi.org/10.1016/03781127(93)90046-P.

Del Valle JI. La ecuacion de crecimiento de Von Bertalanffy en la determinacion de la edad y el crecimiento de arboles tropicales. Revista Facultad Nacional de Agronomia 1986; 39(1): 61-74.

Ebling A, Watzlawick LF, Rodrigues AL, Longhi SJ, Longhi RV, Abrão SF. Acuracidade da distribuição diamétrica entre métodos de projeção em Floresta Ombrófila Mista. Ciência Rural 2012; 42(6): 1020-1026. http://dx.doi.org/10.1590/ S0103-84782012000600011.

Ferreira GWD, Ferraz AC Fo, Pinto ALR, Scolforo JRS. Influência do desbaste na forma do fuste de povoamentos naturais de Eremanthus incanus (Less.) Less. Ciências Agrárias 2014; 35(4): 1707-1720.
Husch B, Beers TW, Kershaw JA Jr. Forest mensuration. 4th ed. New Jersey: John Wiley and Sons; 2003.

Imaña Encinas J, Silva GF, Pinto JRR. Idade e crescimento das árvores. Comunicações Técnicas Florestais 2005; 7(1): 3-19.

Machado VM, Santos JB, Pereira IM, Cabral CM, Lara RO, Amaral CS. Controle químico e mecânico de plantas daninhas em áreas em recuperação. Revista Brasileira de Herbicidas 2012; 11(2): 139-147. http://dx.doi.org/10.7824/ rbh.v11i2.153.

Neves SC, Abreu PAA, Fraga LMS. Fisiografia. In: Silva AC, Pedreira LCVSF, Abreu PAA. Serra do espinhaço meridional, paisagens e ambientes. Belo Horizonte: $\mathrm{O}$ Lutador; 2005.

Pérez JFM, Scolforo JRS, Oliveira AD, Mello JM, Borges LFR, Camolesi JF. Sistema de manejo para a candeia Eremanthus erythropappus (DC.) MacLeish: a opção do sistema de corte seletivo. Cerne 2004; 10(2): 257-273.

$\mathrm{R}$ Development Core Team. R: a language and environment for statistical computing [online]. Vienna: R Foundation for Statistical Computing; 2013 [citado em 2016 abr 20]. Disponível em: http://www.R-project.org/

Scolforo JRS, Oliveira AD, Davide AC. Manejo Sustentável da Candeia: o caminhar de uma nova experiência florestal em Minas Gerais. Lavras: UFLA; 2012.

Scolforo JRS, Pulz FA, Mello JM, Oliveira AT Fo. Modelo de produção para floresta nativa como base para manejo sustentado. Cerne 1996; 2(1): 112-137.

Silva LA, Souza AL, Jesus RM. Ciclo de corte de uma floresta natural secundária por meio da metodologia do tempo de passagem por classes diamétricas sucessivas. Revista Árvore 1999; 23(3): 293-299.

Souza AL, Araújo PA, Campos JCC, Paula F No. Dinâmica de crescimento em diâmetro de uma floresta primária sem interferência: Uma análise pelo tempo de passagem entre classes diamétricas. Revista Árvore 1993; 17(2): 129-145. 